\title{
FLOTAC: a new sensitive technique for the diagnosis of hookworm infections in humans
}

\author{
Jürg Utzinger ${ }^{a, 1}$, Laura Rinaldi ${ }^{\text {b, }}{ }^{\text {, Laurent K. Lohourignon }}$, \\ Fabian Rohner ${ }^{\text {d,e }}$, Michael B. Zimmermann ${ }^{d}$, Andres B. Tschannen ${ }^{e}$, \\ Eliézer K. N'Goran ${ }^{c, e}$, Giuseppe Cringoli ${ }^{\mathrm{a}, *}$
}

\author{
a Department of Public Health and Epidemiology, Swiss Tropical Institute, P.O. Box, CH-4002 Basel, Switzerland \\ b Dipartimento di Patologia e Sanità Animale, Facoltà di Medicina Veterinaria, Università degli Studi di Napoli Federico II, \\ CREMOPAR Regione Campania, Via della Veterinaria 1, 80137 Napoli, Italy \\ ' UFR Biosciences, Université d'Abidjan-Cocody, 22 BP 582, Abidjan 22, Côte d'Ivoire \\ ${ }^{\mathrm{d}}$ Human Nutrition Laboratory, Institute of Food Science and Nutrition, ETH Zurich, Schmelzbergstrasse 7, CH-8092 Zurich, \\ Switzerland \\ e Centre Suisse de Recherches Scientifiques, 01 BP 1303, Abidjan 01, Côte d'Ivoire
}

Received 21 May 2007; received in revised form 7 September 2007; accepted 11 September 2007 Available online 29 October 2007

\author{
KEYWORDS \\ Helminths; \\ Hookworm infections; \\ Diagnosis; \\ Kato-Katz; \\ FLOTAC; \\ Sensitivity; \\ Côte d'Ivoire
}

\begin{abstract}
Summary Hookworms infect more than $10 \%$ of the world's population, but current diagnostic tools have drawbacks. Our objective was to compare the diagnostic performance of three methods (Kato-Katz, ether concentration and FLOTAC techniques) for hookworm diagnosis. Stool samples were obtained from 102 schoolchildren in Côte d'Ivoire. First, a duplicate $41.7 \mathrm{mg}$ Kato-Katz thick smear was prepared. Next, a small portion of stool (mean weight $1.8 \mathrm{~g}$ ) was preserved in sodium acetate-acetic acid-formalin and forwarded to a European laboratory. These samples were split in three parts, one processed by an ether concentration technique and two by the FLOTAC technique. All samples were examined by experienced technicians for hookworm eggs using light microscopy. The observed hookworm prevalences as assessed by the FLOTAC, Kato-Katz and ether concentration techniques were $65.7 \%, 51.0 \%$ and $28.4 \%$, respectively. Considering the combined results as the diagnostic 'gold' standard, the FLOTAC technique had a sensitivity of $88.2 \%$ compared with $68.4 \%$ for the Kato-Katz and $38.2 \%$ for the ether concentration techniques. The Kato-Katz method resulted in a significantly higher mean number of eggs per gram of stool (155.8 EPG) compared with the FLOTAC (37.7 EPG) and ether concentration (5.7 EPG) methods. The FLOTAC method shows promise as an important new tool for individual hookworm diagnosis and for rigorous monitoring of helminth control programmes. [Clinical Trial No. ISRCTN21782274].

(c) 2007 Royal Society of Tropical Medicine and Hygiene. Published by Elsevier Ltd. All rights reserved.
\end{abstract}

* Corresponding author. Tel.: +390812536 283; fax: +39 0812536282.

E-mail address: cringoli@unina.it (G. Cringoli).

1 These authors contributed equally to this work.

0035-9203/\$ - see front matter @ 2007 Royal Society of Tropical Medicine and Hygiene. Published by Elsevier Ltd. All rights reserved. doi:10.1016/j.trstmh.2007.09.009 


\section{Introduction}

There is growing recognition of the huge public health significance of soil-transmitted helminthiases (Bethony et al., 2006; Lammie et al., 2006; WHO, 2002). Two-thirds of the world's population are at risk of contracting an infection with one or more of the main soil-transmitted helminths, including Ascaris lumbricoides, the hookworms (Ancylostoma duodenale and Necator americanus) and Trichuris trichiura (Horton, 2003; Utzinger and Keiser, 2004). The collective burden of soil-transmitted helminthiases may be as high as 39 million disability-adjusted life-years, similar to that due to malaria or tuberculosis (Hotez et al., 2006a; WHO, 2002). Fortunately, local, regional and global commitments to control soil-transmitted helminthiases and other so-called neglected tropical diseases have grown recently, with efforts getting underway to harness potential synergies through the integration of control programmes targeting multiple neglected tropical diseases simultaneously (Brady et al., 2006; Brooker et al., 2006; Fenwick, 2006; Lammie et al., 2006; Molyneux et al., 2005). The main thrust of the new helminth control initiatives is that safe, efficacious and inexpensive drugs are available to reduce the burden caused by these diseases (Hotez et al., 2006a). The WHO recommends treatment of entire communities with anthelminthic drugs in settings where infections are highly prevalent (e.g. $>50 \%$ of school-aged children infected), without prior diagnosis (WHO, 2002, 2006).

The high prevalence of soil-transmitted helminth infections in the tropics and subtropics is explained by climatic factors coupled with poor hygiene standards and the lack of adequate sanitation (de Silva et al., 2003). Soil-transmitted helminth infections were highly prevalent in the southern parts of the USA and elsewhere in the now-developed world less than a century ago (Stiles, 1939; Stoll, 1947). Treatment of infected individuals and improved sanitation together with socioeconomic development have led to the elimination of hookworm disease as a public health problem in the southern USA (Brown, 1976) and may explain the recent declines observed in Southeast Asia (de Silva et al., 2003).

An infection with hookworm can cause abdominal pain and diarrhoea, but the main clinical consequence is iron deficiency anaemia caused by intestinal blood loss resulting from the haematophagy of these worms and from areas of microhaemorrhages on the gut mucosa when the worms detach (Bethony et al., 2006; Brooker et al., 2006; Stoltzfus et al., 1996). The definitive diagnosis of a hookworm infection rests on microscopic visualisation of eggs in stool. Several methods are available for determining the presence and intensity of hookworm infections. Because of its simplicity and relatively low cost, the Kato-Katz technique (Katz et al., 1972) is widely used in epidemiological surveys and is recommended by the WHO for monitoring helminth control programmes (WHO, 1991). The ether-based concentration method (Allen and Ridley, 1970) is also widely used for diagnosis of helminths and intestinal protozoa and has the advantage of fixing the eggs or cysts (Cheesbrough, 2005). The latter issue is important in remote settings where microscopy may not be readily available.

Recent reports suggest that the sensitivity of a single Kato-Katz thick smear for hookworm diagnosis is low, par- ticularly in cases of low infection intensities (Booth et al., 2003; Utzinger et al., 2002). Considering the pooled results from 3578 stool examinations among schoolchildren in Côte d'Ivoire, using the Kato-Katz and ether concentration methods as the diagnostic 'gold' standard, the sensitivities of the Kato-Katz technique and the ether concentration method were $77.8 \%$ and $58.6 \%$, respectively (Raso et al., 2006). Comparison of 988 stool samples from Malawi subjected to the Kato-Katz and ether concentration method revealed respective prevalences of $27.3 \%$ and $19.3 \%$ (Dacombe et al., 2007). Stool samples from 354 Zanzibari infants (aged 5-11 months) were examined by five different methods; the hookworm prevalence based on a duplicate Kato-Katz thick smear was $6.1 \%$, whereas two ether concentration methods revealed prevalences of 5.0\% and 5.8\% (Goodman et al., 2007).

A novel multivalent faecal egg count method, the socalled FLOTAC technique, has been described recently both for human and veterinary medicine (Cringoli, 2006). This method is facilitated by the FLOTAC ${ }^{\circledR}$ apparatus, which has been designed to carry out flotation in a centrifuge, followed by a transversal cut (i.e. translation) of the apical portion of the floating suspension. The FLOTAC technique allows quantification of eggs and/or larvae of nematodes and trematodes as well as cysts and oocysts of intestinal protozoa in up to $1 \mathrm{~g}$ of faeces. It should therefore considerably improve the sensitivity of the aforementioned diagnostic tools. The present paper reports use of the FLOTAC technique for hookworm diagnosis in human stool samples, with results being compared with the standard Kato-Katz and ether concentration methods. Implications and arising research needs are discussed.

\section{Materials and methods}

\subsection{Study area and stool samples}

Stool samples were obtained from schoolchildren aged 6-14 years who were recruited from five primary schools in the district of Toumodi, located approximately $180 \mathrm{~km}$ northwest of Abidjan, the economic capital of Côte d'Ivoire. The region is situated in the 'V-Baoule', the transition zone from rainforest to savannah. The average annual temperature is $27^{\circ} \mathrm{C}$ and the mean annual precipitation is $1060 \mathrm{~mm}$ (N'Goran et al., 1998; Rohner et al., 2007). Helminth infections, particularly hookworms and Schistosoma haematobium, are highly endemic in this part of Côte d'Ivoire (N'Goran et al., 1998, 2001; Rohner et al., 2007).

Stool sample collection was carried out in December 2006, in the middle of the dry season. Overall, 116 schoolchildren were randomly selected from 640 schoolchildren who were found to be mildly to moderately anaemic (defined as haemoglobin level $>80 \mathrm{~g} / \mathrm{l}$ and $<115 \mathrm{~g} / \mathrm{l}$ for children aged $6-12$ years and $>80 \mathrm{~g} / \mathrm{l}$ and $<120 \mathrm{~g} / \mathrm{l}$ in children 12-14 years).

\subsection{Ethical considerations}

The study was embedded in a randomised controlled trial (No. ISRCTN21782274) assessing the effect of different interventions for anaemia prevention, and the stool collection 
reported here was done during the baseline survey. Written informed consent was obtained from the parents or legal guardians of participating children. At the end of the trial, all children with a hookworm or other soil-transmitted helminth infection were treated with a single $500 \mathrm{mg}$ oral dose of mebendazole.

\subsection{Field and laboratory procedures}

Stool samples were transferred to the nearby laboratory and processed as follows. First, a duplicate Kato-Katz thick smear was prepared from each stool sample on microscope slides using $41.7 \mathrm{mg}$ punched plastic templates (Katz et al., 1972). After a clearing time of 30-45 min, the slides were examined under a light microscope by experienced laboratory technicians. The numbers of hookworm eggs and other soil-transmitted helminths (i.e. A. lumbricoides and T. trichiura) were counted and recorded separately. For quality control, $10 \%$ of the slides were re-examined by the senior technician. The mean number of hookworm eggs was calculated from the two slides examined per child and was multiplied by a factor 24 to obtain the number of eggs per gram of stool (EPG).

Second, a hazelnut-sized portion of each stool sample was placed into a small tube containing $10 \mathrm{ml}$ of sodium acetate-acetic acid-formalin (SAF) solution (Bogoch et al., 2006; Marti and Escher, 1990). Each aliquot was weighed (PB303-S Delta Range; Mettler Toledo, Greifensee, Switzerland). The SAF-fixed stool samples were forwarded to the Laboratory of Veterinary Parasitology, University of Naples 'Federico II' (Naples, Italy) and simultaneously processed by an ether concentration method (Allen and Ridley, 1970) and the FLOTAC technique (Cringoli, 2006). Once at the laboratory, 25 small glass balls were put into each SAF-fixed stool sample and the tubes were vigorously shaken until all the faecal matter was broken down. Each mixture was poured through a wire mesh screen with an aperture of $350 \mu \mathrm{m}$ and the strained suspension was equally split into three tubes.

Each tube (containing one-third of the original weight of each stool sample) was centrifuged for $1 \mathrm{~min}$ at $2000 \mathrm{rpm}$ and then randomly assigned to a method. Specifically, the first tube was used to perform the ether concentration method (Allen and Ridley, 1970), whose resulting slides were examined under a light microscope for the presence of helminths by experienced laboratory technicians. The remaining two tubes were filled to $5 \mathrm{ml}$ with the following two flotation solutions: (i) sodium nitrate (specific gravity $=1.20$ ) for detection and counting of nematode eggs (e.g. hookworms) and eggs of cestodes; and (ii) zinc sulphate plus potassium iodomercurate (specific gravity $=1.45$ ) for detection and counting of nematode larvae (e.g. Strongyloides stercoralis) and trematode eggs (e.g. Schistosoma mansoni and Fasciola hepatica). These two flotation solutions were chosen from a battery of 14 solutions with specific gravities ranging between 1.20 and 1.45 (Cringoli et al., 2004), after pre-testing with eight stool samples selected at random. Egg counts commenced following centrifugation ( $5 \mathrm{~min}$ at $1000 \mathrm{rpm}$ ) and translation, and they were performed under both grids (volume of each $=5 \mathrm{ml}$ ), corresponding to each of the two flotation solutions used (Cringoli, 2006).
For both methods, EPG was calculated as follows: $E P G=$ no. of eggs counted/weight of stool sample in $g \times 3$.

\subsection{Statistical analyses}

Data were double-entered and cross-checked and statistical analyses were performed using version 13 of SPSS software for Windows (SPSS Inc., Chicago, IL, USA). The final analysis focused on those stool samples where complete data records (i.e. Kato-Katz, ether concentration and FLOTAC results) were available.

Agreement between two methods (i.e. Kato-Katz versus FLOTAC, ether concentration versus FLOTAC, and Kato-Katz versus ether concentration) for hookworm diagnosis was assessed using the $\kappa$ statistic (Thrusfield, 1995). The sensitivity (proportion of true positives in the population) and negative predictive value (proportion of individuals with a negative test result who are correctly diagnosed) were calculated for each of the three methods, considering the combined results from the individual methods as the diagnostic 'gold' standard. This approach maximised sensitivity values, which is argued as follows: the detection of a hookworm egg by any of the methods employed was considered as a true-positive result. Following this approach, however, specificity and positive predictive values could not be calculated (Bogoch et al., 2006). Furthermore, mean, standard error (SE), and 25th, 50th and 75th percentiles of EPG values were calculated for the three different techniques. Since EPG values were overdispersed, as revealed by analysis of the normality tests of Shapiro-Wilk $(P<0.05)$ and the normal Q-Q plots, the values were transformed utilising the natural logarithm $(\ln )$ of EPG +1 . The reason for adding 1 to each EPG value is to avoid the mathematically non-defined logarithm of zero. Statistical differences between the mean EPG values (using the In-transformed data) were analysed using ANOVA, in conjunction with the Bonferroni test for post-hoc comparison.

\section{Results}

\subsection{Operational results}

Overall, 116 stool samples were collected and duplicate Kato-Katz thick smear results were available from 115 of them. Sufficient quantities were available to preserve a small portion in SAF solution from 110 stool samples. These SAF-preserved samples were forwarded to Italy, where, as mentioned previously, eight samples were used for identification of the most suitable flotation solutions for the FLOTAC technique. Complete data records were available for 102 stool samples; all subsequent analyses pertain to this cohort. The mean weight of the SAF-preserved stool samples was $1.80 \mathrm{~g}$ (range $1.00-2.18 \mathrm{~g}$ ).

\subsection{Comparison of methods}

Tables 1-3 show the results of the comparison between the different diagnostic approaches by means of two-way contingency tables of frequencies. Agreements between two methods compared with each other are reported, including $\kappa$ statistics. Of the 102 stool samples examined for hook- 
Table 1 Two-way contingency table of frequencies showing agreement between the Kato-Katz and FLOTAC techniques for the diagnosis of hookworms in human stool samples ${ }^{a}$

\begin{tabular}{lllr}
\hline FLOTAC & \multicolumn{2}{l}{ Kato-Katz } & \\
\cline { 2 - 4 } & Positive & Negative & Total \\
\hline Positive & 43 & 24 & 67 \\
Negative & 9 & 26 & 35 \\
Total & 52 & 50 & 102 \\
\hline
\end{tabular}

a $\kappa$ agreement $=0.349(P<0.001)$.

Table 2 Two-way contingency table of frequencies showing agreement between the ether concentration and FLOTAC techniques for the diagnosis of hookworms in human stool samples $^{\mathrm{a}}$

\begin{tabular}{lllr}
\hline FLOTAC & \multicolumn{3}{l}{ Ether concentration } \\
\cline { 2 - 4 } & Positive & Negative & Total \\
\hline Positive & 29 & 38 & 67 \\
Negative & 0 & 35 & 35 \\
Total & 29 & 73 & 102 \\
\hline
\end{tabular}

a $\kappa$ agreement $=0.344(P<0.001)$.

Table 3 Two-way contingency table of frequencies showing agreement between the Kato-Katz and ether concentration techniques for the diagnosis of hookworms in human stool samples $^{\mathrm{a}}$

\begin{tabular}{|c|c|c|c|}
\hline \multirow[t]{2}{*}{ Ether concentration } & \multicolumn{3}{|c|}{ Kato-Katz } \\
\hline & Positive & Negative & Total \\
\hline Positive & 21 & 8 & 29 \\
\hline Negative & 31 & 42 & 73 \\
\hline Total & 52 & 50 & 102 \\
\hline
\end{tabular}

a $\kappa$ agreement $=0.242(P=0.006)$.

worms, 67 (65.7\%, 95\% confidence interval (CI) 55.5-74.6\%) resulted positive when examined with the FLOTAC technique, 52 (51.0\%, 95\% Cl 40.9-60.9\%) after examination of two Kato-Katz thick smears derived from the same stool samples, and 29 (28.4\%, 95\% Cl 20.2-38.4\%) based on the ether concentration method. According to previously proposed benchmarks (Thrusfield, 1995), agreement between the FLOTAC and Kato-Katz techniques was only 'fair' (0.349). A similar and therefore only fair agreement
Table 5 Hookworm eggs per gram of stool (EPG) (arithmetic mean, standard error (SE) and percentiles) detected by the Kato-Katz, FLOTAC and ether concentration techniques

\begin{tabular}{lrrlcr}
\hline Technique & Mean & SE & \multicolumn{4}{l}{ Percentiles } \\
\cline { 3 - 6 } & & & 25th & 50th & 75th \\
\hline Kato-Katz & 155.8 & 41.9 & 0 & 12.0 & 108.0 \\
FLOTAC & 37.7 & 16.1 & 0 & 0 & 1.6 \\
Ether concentration & 5.7 & 2.2 & 0 & 3.2 & 17.2 \\
\hline
\end{tabular}

was found between FLOTAC and the ether concentration method (0.344). An even weaker agreement was found between the Kato-Katz and ether concentration methods (0.242).

The pooled data revealed an overall hookworm prevalence of $74.5 \%(95 \% \mathrm{Cl} 64.7-82.4 \%)$. The sensitivity and negative predictive values of the individual methods, compared with the pooled results, which was considered our diagnostic 'gold' standard, are summarised in Table 4. The sensitivities of the FLOTAC technique, the duplicate Kato-Katz thick smears and the ether-based concentration method were $88.2 \%(95 \% \mathrm{Cl} 78.2-94.1 \%), 68.4 \%(95 \% \mathrm{Cl}$ $56.6-78.3 \%)$ and $38.2 \%(95 \% \mathrm{Cl} 27.5-50.1 \%)$, respectively.

Table 5 summarises hookworm EPG values (mean, SE and percentiles) according to the diagnostic approach taken. The mean EPG revealed by the FLOTAC (37.7) and Kato-Katz (155.8) techniques were significantly higher than that obtained by the ether concentration method (5.7) ( $P<0.001$ for both comparisons). The mean EPG produced by the Kato-Katz technique was higher than that produced by FLOTAC and the difference showed statistical significance $(P=0.042)$.

Other helminth eggs (i.e. A. lumbricoides and T. trichiura) and helminth larvae (e.g. S. stercoralis) were also detected in the stool samples examined; however, these results were not included in the present paper because of very low observed prevalences. No S. mansoni eggs were detected using all three methods considered.

\section{Discussion}

Notwithstanding significant advances in our understanding of the epidemiology of hookworm infections, growing appreciation of the 'true' public health impact of this neglected tropical disease, development of new tools and progress made in the control of hookworm infection in different settings (Brooker et al., 2004, 2006; Hotez et al., 2006b), little

Table 4 Sensitivity and negative predictive values (NPV) of the FLOTAC, Kato-Katz and ether concentration techniques for the diagnosis of hookworms in human stool samples when considering the pooled results from the three methods as the diagnostic 'gold' standard

\begin{tabular}{llr}
\hline Technique & Sensitivity $(95 \% \mathrm{Cl})$ & NPV (95\% Cl) \\
\hline FLOTAC & $88.2 \%(78.2-94.1 \%)$ & $79.5 \%(64.2-89.7 \%)$ \\
Kato-Katz & $68.4 \%(56.6-78.3 \%)$ & $67.6 \%(55.6-77.7 \%)$ \\
Ether concentration & $38.2 \%(27.5-50.1 \%)$ & $60.8 \%(51.5-69.5 \%)$ \\
\hline
\end{tabular}


emphasis has been placed on the development of novel diagnostics or the standardisation of existing copromicroscopic techniques. Our findings provide important new information on the performance of available methods for the in vivo diagnosis of hookworm infection in humans. Strengths and weaknesses of the different methods are discussed, along with implications and arising research needs.

The first issue pertains to sensitivity. Whilst the FLOTAC technique identified 67 of the 102 stool samples as hookworm-positive, a duplicate Kato-Katz thick smear reading and an ether concentration method found 52 and 29 of the samples to be positive, respectively. Thus, the observed prevalence of hookworm infection based on the FLOTAC technique was $29 \%$ higher compared with the Kato-Katz technique and $131 \%$ in the case of the ether concentration method. It follows that the sensitivity of the FLOTAC technique for hookworm diagnosis was considerably higher compared with the other two diagnostic approaches. Importantly, in 38 cases FLOTAC was positive for hookworms whereas the ether concentration method gave a negative result, and 24 times the FLOTAC technique found hookworm eggs whereas the Kato-Katz thick smear readings were negative. All of the 29 hookworm cases diagnosed by the ether concentration method were also found to be positive by FLOTAC. With regard to hookworm-positive Kato-Katz thick smear readings, there were nine cases where FLOTAC produced a negative result. Interestingly, in these cases one out of the two Kato-Katz readings was negative whereas only a few eggs (i.e. 1-3) were found on the other slide, suggesting that only light hookworm infections were missed by FLOTAC. This observation might be explained by the following reasons. First, we used only one of the two chambers of the FLOTAC $^{\circledR}$ apparatus for the diagnosis of hookworms (the other was used for diagnosis of trematodes). Second, the hookworm eggs are very fragile. Results of a recent study performed on faecal samples obtained from dogs, comparing either fresh or SAF-preserved stool samples, showed that in the SAF-conserved samples more than $30 \%$ of hookworm eggs were broken or altered and hence not detected (unpublished data). Third, the highly aggregated distribution of helminth eggs in stool samples may lead to the absence of eggs in cases where only one small sample is examined (Anderson and Schad, 1985; Utzinger et al., 2002).

Our results thus underscore previous observations made for various parasites of veterinary importance: FLOTAC has a higher sensitivity than other more widely used diagnostic approaches (Cringoli, 2006; Rinaldi et al., 2007). Moreover, our study confirms that the prevalence of human hookworm infections, and conceivably also other soil-transmitted helminths and S. mansoni, may be underestimated by routinely used techniques such as the Kato-Katz or ether concentration methods. An important explanation is the small amount of faecal matter analysed by the former method; $41.7 \mathrm{mg}$ of stool in a single Kato-Katz thick smear reading (Katz et al., 1972). Examination of multiple Kato-Katz thick smears from one or ideally from several stool specimens enhances the sensitivity of helminth diagnosis but is operationally challenging, costly and more time consuming (Booth et al., 2003; Engels et al., 1996; Raso et al., 2006; Utzinger et al., 2002). The FLOTAC technique examines a larger amount of faeces. In the present study, a mean of $0.6 \mathrm{~g}$ of stool was subjected to hookworm diagnosis by FLOTAC, but the amount could be doubled (using both chambers) or even higher. Future studies should investigate whether indeed the sensitivity can be further enhanced by examination of larger stool samples.

The second issue pertains to infection intensity. The mean hookworm EPG among children according to the Kato-Katz results (155.8 EPG, i.e. a mean of 6 eggs counted per microscope slide multiplied by a factor of 24 to obtain the number of EPG) was significantly higher when compared with the FLOTAC technique (37.7 EPG, i.e. a mean of 23 eggs counted in the apparatus multiplied by a factor of 1.4-3). At first sight, this result raises concern about the ability of the FLOTAC method for quantification of infection intensity. Different factors might have contributed to the discrepancy of EPG between Kato-Katz and FLOTAC. Importantly, it has been shown before that the observed helminth infection intensity is a function of sampling effort; the more samples subjected to the Kato-Katz method, the lower the infection intensity. For example, the mean S. mansoni egg count among 253 schoolchildren from Côte d'Ivoire was 161 EPG based on a single Kato-Katz thick smear, whereas the observed infection intensity was 111 EPG after examination of four Kato-Katz thick smears derived from different stool samples (Utzinger et al., 2000). Whilst this issue actually challenges the accuracy of the Kato-Katz method for estimating helminth infection intensity, the point mentioned before that preservation of the stool samples in SAF might result in some hookworm eggs being broken or altered suggests that the mean EPG has been underestimated. This issue should be addressed in future investigations comparing mean EPG revealed by FLOTAC using either fresh or SAFconserved stool samples.

It is important to note that the Kato-Katz technique and the FLOTAC method resulted in significantly higher EPG values than the ether concentration method (5.7 EPG). A 27.3-fold difference was found between the Kato-Katz and ether concentration methods, whereas the corresponding factor between FLOTAC and the ether concentration method was 6.6. Given the low sensitivity and the significant underestimation of infection intensity by the ether concentration method, this technique when used alone should be discouraged for hookworm diagnosis.

If the high sensitivity of the FLOTAC technique is confirmed by other groups in different settings and further improvements are made with regard to quantifying infection intensity (e.g. studies on fixatives), widespread use of the Kato-Katz technique for epidemiological surveys and monitoring of helminth control programmes is put in question. Particularly in settings where the overall prevalences and intensities of helminth infections are low, for example after repeated rounds of chemotherapy, results obtained by the Kato-Katz method must be analysed with care. New data suggest that the Kato-Katz technique has drawbacks for screening stool samples from infants, as helminth infection intensities tend to be low and stool samples are more liquid than those collected from school-aged children or adults (Goodman et al., 2007). This issue is of considerable relevance, as recent studies suggest that infants and pre-school children are at higher risk of helminth infections and associated morbidity than previously assumed (Goodman et al., 2007; Stothard and Gabrielli, 2007). 
Three further aspects are worth highlighting. First, since the FLOTAC technique is able to examine stool quantities up to $1 \mathrm{~g}$ or more, there is no need for high multiplication factors for determining EPG. In the present study, the mean weight of stool samples subjected to hookworm diagnosis by FLOTAC was $0.6 \mathrm{~g}$, and hence a multiplication factor ranging between 1.4 and 3 was used. Note that for a single Kato-Katz thick smear reading, the corresponding multiplication factor for the EPG calculation is 24, or in other words 8-17 times higher than for the FLOTAC technique. The negative implications of high multiplication factors for EPG calculations have been discussed elsewhere (Cringoli et al., 2004; Mes et al., 2001). Second, the FLOTAC technique is multivalent. Hence, the technique can be used for the simultaneous diagnosis of different helminths as well as intestinal protozoa. This issue is of relevance because multiple-species parasitic infections are the rule rather than the exception in the developing world and hence a combination of different diagnostic approaches is recommended (Brooker and Utzinger, 2007; Raso et al., 2004). Third, subsequent studies should address the feasibility of the FLOTAC technique when applied in epidemiological studies in resource-poor settings, including technicians' satisfaction with this method and issues of costs and cost effectiveness.

In conclusion, our data suggest that the FLOTAC technique may improve the ability to diagnose human hookworm infections accurately. These findings need to be confirmed in other epidemiological settings where hookworm, other soil-transmitted helminths, S. mansoni and intestinal protozoa are prevalent. The FLOTAC method shows promise as an important new tool for individual hookworm diagnosis and for rigorous monitoring of helminth control programmes.

Authors' contributions: JU, LR, MBZ and GC designed the study protocol; LKL, FR, ABT and EKN designed and carried out the field work; LKL prepared the stool specimens for the Kato-Katz and the ether concentration methods and examined the slides under a light microscope; LR, LKL and GC prepared the stool specimens for the FLOTAC method and examined the slides; JU, LR and GC drafted the manuscript. All authors read, revised and approved the final manuscript. $\mathrm{GC}$ is guarantor of the paper.

Acknowledgements: We thank the village authorities, teachers and participating schoolchildren from Aluminankro, Bringakro, Gbohua, N'da Kouassikro and Tafissou in the district of Toumodi, as well as the laboratory technicians Mamadou Traoré and Sabrina Carbone for expert help in the field and behind the bench. We thank Giuseppe and Massimo Federico for their enthusiastic support in assisting the development of the FLOTAC apparatus. The comments from two anonymous referees helped to improve the manuscript further.

Funding: Swiss Federal Institute of Technology and the Hochstrasser Foundation. J. Utzinger is grateful to the Swiss National Science Foundation for sustained financial support (project no. PPOOB-102883).

Conflicts of interest: The FLOTAC apparatus has been developed and is patented by $\mathrm{G}$. Cringoli, but the patent will be handed over to the University of Naples 'Federico II' shortly. At present, the method is being validated by different research groups focusing on human and veterinary parasitology. Should these validations be successful, the method will be provided free of charge to public research centres, including the $\mathrm{WHO}$ and universities. The funding agencies cited above and the fact that one of the authors is the current patent holder of the FLOTAC apparatus played no role in the design or implementation of the study reported, analysis of the data, or the preparation, submission and revision of the manuscript. All other authors have no conflicts of interest concerning the work reported in this paper.

Ethical approval: The study was embedded in a randomised controlled trial entitled 'Iron fortification and parasite control to reduce anaemia among schoolchildren in Côte d'Ivoire', which was approved by the institutional review boards of the Swiss Federal Institute of Technology (Zurich, Switzerland) and the Centre Suisse de Recherches Scientifiques (Abidjan, Côte d'Ivoire). The global study received ethical clearance from the Ethics Committee of the University and the State of Basel (reference number 224/06) and the 'Comité National d'Ethique des Sciences de la Vie et de la Santé' (reference number MSHP/CAB/CNESVS/06). The trial is registered as an International Standard Randomised Controlled Trial (reference number ISRCTN21782274).

\section{References}

Allen, A.V.H., Ridley, D.S., 1970. Further observations on the formol-ether concentration technique for faecal parasites. J. Clin. Pathol. 23, 545-546.

Anderson, R.M., Schad, G.A., 1985. Hookworm burdens and faecal egg counts: an analysis of the biological basis of variation. Trans. R. Soc. Trop. Med. Hyg. 79, 812-825.

Bethony, J., Brooker, S., Albonico, M., Geiger, S.M., Loukas, A., Diemert, D., Hotez, P.J., 2006. Soil-transmitted helminth infections: ascariasis, trichuriasis, and hookworm. Lancet 367, 1521-1532.

Bogoch, I.I., Raso, G., N'Goran, E.K., Marti, H.P., Utzinger, J., 2006. Differences in microscopic diagnosis of helminths and intestinal protozoa among diagnostic centres. Eur. J. Clin. Microbiol. Infect. Dis. 25, 344-347.

Booth, M., Vounatsou, P., N'Goran, E.K., Tanner, M., Utzinger, J., 2003. The influence of sampling effort and the performance of the Kato-Katz technique in diagnosing Schistosoma mansoni and hookworm co-infections in rural Côte d'Ivoire. Parasitology 127 , 525-531.

Brady, M.A., Hooper, P.J., Ottesen, E.A., 2006. Projected benefits from integrating NTD programs in sub-Saharan Africa. Trends Parasitol. 22, 285-291.

Brooker, S., Utzinger, J., 2007. Integrated disease mapping in a polyparasitic world. Geospatial Health 1, 141-146.

Brooker, S., Bethony, J., Hotez, P.J., 2004. Human hookworm infection in the 21st century. Adv. Parasitol. 58, 197-288.

Brooker, S., Clements, A.C.A., Bundy, D.A.P., 2006. Global epidemiology, ecology and control of soil-transmitted helminth infections. Adv. Parasitol. 62, 221-261.

Brown, E.R., 1976. Public health in imperialism: early Rockefeller programs at home and abroad. Am. J. Public Health 66, 897903.

Cheesbrough, M. (Ed.), 2005. Parasitological tests, in: District Laboratory Practice in Tropical Countries. Tropical Health Technologies, Cambridge, pp. 178-306. 
Cringoli, G., 2006. FLOTAC, a novel apparatus for a multivalent faecal egg count technique. Parassitologia 48, 381-384.

Cringoli, G., Rinaldi, L., Veneziano, V., Capelli, G., Scala, A., 2004. The influence of flotation solution, sample dilution and the choice of McMaster slide area (volume) on the reliability of the McMaster technique in estimating the faecal egg counts of gastrointestinal strongyles and Dicrocoelium dendriticum in sheep. Vet. Parasitol. 123, 121-131.

Dacombe, R.J., Crampin, A.C., Floyd, S., Randall, A., Ndhlovu, R., Bickle, Q., Fine, P.E., 2007. Time delays between patient and laboratory selectively affect accuracy of helminth diagnosis. Trans. R. Soc. Trop. Med. Hyg. 101, 140-145.

de Silva, N.R., Brooker, S., Hotez, P.J., Montresor, A., Engels, D., Savioli, L., 2003. Soil-transmitted helminth infections: updating the global picture. Trends Parasitol. 19, 547-551.

Engels, D., Sinzinkayo, E., Gryseels, B., 1996. Day-to-day egg count fluctuation in Schistosoma mansoni infection and its operational implications. Am. J. Trop. Med. Hyg. 54, 319-324.

Fenwick, A., 2006. New initiatives against Africa's worms. Trans. R. Soc. Trop. Med. Hyg. 100, 200-207.

Goodman, D., Haji, H.J., Bickle, Q.D., Stoltzfus, R.J., Tielsch, J.M., Ramsan, M., Savioli, L., Albonico, M., 2007. A comparison of methods for detecting the eggs of Ascaris, Trichuris, and hookworm in infant stool, and the epidemiology of infection in Zanzibari infants. Am. J. Trop. Med. Hyg. 76, 725-731.

Horton, J., 2003. Human gastrointestinal helminth infections: are they now neglected diseases? Trends Parasitol. 19, 527-531.

Hotez, P.J., Molyneux, D.H., Fenwick, A., Ottesen, E., Ehrlich Sachs, S., Sachs, J.D., 2006a. Incorporating a rapid-impact package for neglected tropical diseases with programs for HIV/AIDS, tuberculosis, and malaria. PLoS Med. 3, e102.

Hotez, P.J., Bethony, J., Bottazzi, M.E., Brooker, S., Diemert, D., Loukas, A., 2006b. New technologies for the control of human hookworm infection. Trends Parasitol. 22, 327331.

Katz, N., Chaves, A., Pellegrino, J., 1972. A simple device for quantitative stool thick-smear technique in schistosomiasis mansoni. Rev. Inst. Med. Trop. Sao Paulo 14, 397-400.

Lammie, P.J., Fenwick, A., Utzinger, J., 2006. A blueprint for success: integration of neglected tropical disease control programmes. Trends Parasitol. 22, 313-321.

Marti, H., Escher, E., 1990. SAF-an alternative fixation solution for parasitological stool specimens. Schweiz. Med. Wochenschr. 120, 1473-1476 [in German].

Mes, T.H.M., Ploeger, H.W., Terlou, M., Kooyman, F.N.J., Van der Ploeg, M.P.J., Eysker, M., 2001. A novel method for the isolation of gastro-intestinal nematode eggs that allows automated analysis of digital images of egg preparations and high throughput screening. Parasitology 123, 309-314.

Molyneux, D.H., Hotez, P.J., Fenwick, A., 2005. 'Rapid-impact interventions': how a policy of integrated control for Africa's neglected tropical diseases could benefit the poor. PLoS Med. 2, e336.

N'Goran, E.K., Utzinger, J., Traore, M., Lengeler, C., Tanner, M., 1998. Use of a questionnaire for quick identification of the principal foci of urinary bilharziasis in central Ivory Coast. Med. Trop. (Mars) 58, 253-260 [in French].
N'Goran, E.K., Utzinger, J., N'Guessan, A.N., Müller, I., Zamblé, K., Lohourignon, K.L., Traoré, M., Sosthène, B.A., Lengeler, C., Tanner, M., 2001. Reinfection with Schistosoma haematobium following school-based chemotherapy with praziquantel in four highly endemic villages in Côte d'Ivoire. Trop. Med. Int. Health 6, 817-825.

Raso, G., Luginbühl, A., Adjoua, C.A., Tian-Bi, N.T., Silué, K.D., Matthys, B., Vounatsou, P., Wang, Y., Dumas, M.E., Holmes, E., Singer, B.H., Tanner, M., N'Goran, E.K., Utzinger, J., 2004. Multiple parasite infections and their relationship to self-reported morbidity in a community of rural Côte d'Ivoire. Int. J. Epidemiol. 33, 1092-1102.

Raso, G., Vounatsou, P., Gosoniu, L., Tanner, M., N'Goran, E.K., Utzinger, J., 2006. Risk factors and spatial patterns of hookworm infection among schoolchildren in a rural area of western Côte d'Ivoire. Int. J. Parasitol. 36, 201-210.

Rinaldi, L., Russo, T., Schioppi, M., Pennacchio, S., Cringoli, G., 2007. Passalurus ambiguus: new insights into copromicroscopic diagnosis and circadian rhythm of egg excretion. Parasitol. Res. 101, 557-561.

Rohner, F., Zimmermann, M.B., Wegmueller, R., Tschannen, A.B., Hurrell, R.F., 2007. Mild riboflavin deficiency is highly prevalent in school-age children but does not increase risk for anaemia in Côte d'Ivoire. Br. J. Nutr. 97, 970-976.

Stiles, C.W., 1939. Early history, in part esoteric, of the hookworm (uncinariasis) campaign in our southern United States. J. Parasitol. 25, 283-308.

Stoll, N.R., 1947. This wormy world. J. Parasitol. 33, 1-18.

Stoltzfus, R.J., Albonico, M., Chwaya, H.M., Savioli, L., Tielsch, J., Schulze, K., Yip, R., 1996. Hemoquant determination of hookworm-related blood loss and its role in iron deficiency in African children. Am. J. Trop. Med. Hyg. 55, 399-404.

Stothard, J.R., Gabrielli, A.F., 2007. Schistosomiasis in African infants and preschool children: to treat or not to treat? Trends Parasitol. 23, 83-86.

Thrusfield, M., 1995. Veterinary Epidemiology. Blackwell, London.

Utzinger, J., Keiser, J., 2004. Schistosomiasis and soil-transmitted helminthiasis: common drugs for treatment and control. Expert Opin. Pharmacother. 5, 263-285.

Utzinger, J., N'Goran, E.K., N'Dri, A., Lengeler, C., Tanner, M., 2000. Efficacy of praziquantel against Schistosoma mansoni with particular consideration for intensity of infection. Trop. Med. Int. Health 5, 771-778.

Utzinger, J., Vounatsou, P., N'Goran, E.K., Tanner, M., Booth, M., 2002. Reduction in the prevalence and intensity of hookworm infections after praziquantel treatment for schistosomiasis infection. Int. J. Parasitol. 32, 759-765.

WHO, 1991. Basic Laboratory Methods in Medical Parasitology. World Health Organization, Geneva.

WHO, 2002. Prevention and Control of Schistosomiasis and SoilTransmitted Helminthiasis: Report of a WHO Expert Committee. World Health Organization, Geneva, Technical Report Series No. 912.

WHO, 2006. Preventive Chemotherapy in Human Helminthiasis: coordinated use of anthelminthic drugs in control interventions: a manual for health professionals and programme managers. World Health Organization, Geneva. 